\title{
DOG1 and PKC- $\theta$ are useful in the diagnosis of KIT-negative gastrointestinal stromal tumors
}

\author{
Gu-Hyun Kang ${ }^{1}$, Amitabh Srivastava ${ }^{2}$, Young Eun Kim ${ }^{1}$, Hee-Jung Park ${ }^{1}$, Cheol Keun Park ${ }^{1}$, \\ Tae Sung Sohn ${ }^{3}$, Sung Kim ${ }^{3}$, Dae Young Kang ${ }^{4}$ and Kyoung-Mee Kim ${ }^{1}$ \\ ${ }^{1}$ Department of Pathology, Samsung Medical Center, Sungkyunkwan University School of Medicine, Seoul, \\ Korea; ${ }^{2}$ Department of Pathology, Dartmouth-Hitchcock Medical Center, Dartmouth Medical School, \\ Lebanon, NH, USA; ${ }^{3}$ Department of Surgery, Samsung Medical Center, Sungkyunkwan University School of \\ Medicine, Seoul, Korea and ${ }^{4}$ Department of Pathology, Chungnam National University School of Medicine, \\ Chungnam National University Hospital, Daejeon, Korea
}

\begin{abstract}
Pathological diagnosis of gastrointestinal stromal tumors (GISTs) is based on histological findings and immunohistochemical demonstration of the KIT protein. KIT-negative GISTs account for $\sim 5 \%$ of cases and cause diagnostic difficulties. In the era of imatinib therapy, a correct diagnosis of GISTs is important for therapeutic reasons regardless of KIT expression. Recently, DOG1 has been introduced as an important diagnostic marker with high sensitivity and specificity. In this study, immunohistochemical staining for DOG1 and protein kinase C- $\theta$ (PKC- $\theta$ ) in whole tissue sections, and mutation analyses for KIT and PDGFRA were performed in 26 KIT-negative GISTs. Tissue microarrays of $112 \mathrm{KIT}$-positive GISTs were used as controls. Overall, 25 KIT-negative GISTs were located in the stomach, and 1 in the rectum. The histological subtype was spindle in 12, epithelioid in 11, and mixed in 3 cases. The expression of DOG1 and PKC- $\theta$ was positive in 24 $(92 \%)$ and in 25 cases (96\%), respectively. All 26 KIT-negative GISTs expressed either DOG1 or PKC- $\theta$, and 23 cases $(89 \%)$ were positive for both makers. PKC- $\theta$ was positive in two cases (8\%), which lacked both KIT and DOG1 expressions. Mutation analysis showed PDGFRA exon 18 mutation in 15 cases $(58 \%)$ and $K I T$ exon 11 mutation in 1 case $(4 \%)$, whereas the remaining 10 cases $(39 \%)$ were wild type for both KIT and PDGFRA. The expression of DOG1 and PKC- $\theta$ showed no significant difference in KIT-negative and KIT-positive GISTs $(P=1.000$ and $P=0.167$, respectively). Our findings suggest that both DOG1 and PKC- $\theta$ can be used in the diagnosis of KIT-negative GISTs and they show positive staining even in KIT-negative tumors, which are wild type for KIT and PDGFRA on mutation analysis.
\end{abstract}

Modern Pathology (2011) 24, 866-875; doi:10.1038/modpathol.2011.11; published online 25 February 2011

Keywords: diagnosis; DOG1; gastrointestinal stromal tumor; KIT; PKC- $\theta$; therapy

KIT (CD117), an immunohistochemical marker constantly expressed in normal interstitial cells of Cajal, is demonstrable in the vast majority of gastrointestinal stromal tumors (GISTs). However, approximately $4-5 \%$ of GISTs lack KIT expression despite typical clinicopathological features. ${ }^{1-3}$ These tumors have a predilection for the stomach or omentum, and are commonly of the epithelioid or mixed histological type. ${ }^{3-5}$ The lack of KIT expression is

Correspondence: Dr K-M Kim, MD, PhD, Department of Pathology, Samsung Medical Center, Sungkyunkwan University School of Medicine, 50 Ilwon-dong, Gangnam-gu, Seoul 135-710, Korea. E-mail: kkmkys@skku.edu

Received 23 August 2010; revised 11 November 2010; accepted 11 November 2010; published online 25 February 2011 usually found in GISTs that are wild type for KIT and PDGFRA, but is also reported in tumors harboring mutations in exons 9 and 11 of $K I T .^{3,6}$ In rare cases, they cease to express the KIT protein because of clonal evolution after imatinib therapy., Therefore, it is important to find markers that reliably stain GISTs, which are weak or negative for the KIT protein.

Most GISTs contain oncogenic gain-of-function receptor tyrosine kinase mutations involving KIT $(\sim 85 \%)$ or PDGFRA $(\sim 5 \%) .^{9,10}$ Inhibition of constitutively activated tyrosine kinases confers clinical responsiveness in advanced GISTs. ${ }^{11}$ In addition, the type of mutation in hot-spot regions of KIT or PDGFRA has relevance to prognosis and the likelihood of responsiveness to tyrosine kinase 
inhibitors. ${ }^{10,12}$ With growing effectiveness and availability of targeted therapies in GISTs, a precise diagnosis is imperative. ${ }^{13,14}$

DOG1 was found to be selectively expressed in GISTs using gene expression profiling studies, although it is not related to oncogenic activation of KIT and PDGRFA. ${ }^{15}$ It is a protein encoded by TMEM16A (synonyms include TMEM16, FLJ10261, ANO1, ORAOV2, and TAOS2), which is located within the CCND1-EMS1 locus on the human chromosome $11 \mathrm{q} 13 .{ }^{16}$ On the basis of gene expression profile using cDNA microarrays, TMEM16A was found to be upregulated in both KIT and PDGFRA mutant GISTs. ${ }^{15}$ Recently, the mouse monoclonal antibody DOG1.1 was introduced as a sensitive diagnostic marker for GISTs with the additional benefit of detecting about one-third of KIT-negative GISTs. ${ }^{8}$ It was found to be highly expressed in KIT mutant GISTs and in unusual subtypes lacking KIT or PDGFRA mutations. ${ }^{8}$ Moreover, nearly half of KIT-negative GISTs were positive for a new DOG1 antibody (clone K9) on multi-tumor paraffin blocks. ${ }^{5}$ Although DOG1 stains about onethird to half of KIT-negative GISTs, the remaining tumors with typical morphological features of GISTs are difficult to validate immunohistochemically. ${ }^{5,8}$ Depending on the DOG1 antibody used, staining in the normal gastric epithelium, carcinomas, germ cell tumors, melanomas, and rarely in some mesenchymal tumors has also been reported. ${ }^{5,17}$
Protein kinase C- $\theta$ (PKC- $\theta$ ), a member of the PKC family, is expressed in virtually all GISTs and is shown to be very specific by western blots. ${ }^{18}$ Several studies have been reported on the utility of PKC- $\theta$ in identifying KIT-negative GISTs and in discriminating this subset of GISTs from other soft tissue tumors. ${ }^{18-21}$ However, high background staining and expression in schwannomas have limited its diagnostic utility. ${ }^{2}$ In our experience, an optimal setting of the staining conditions can remove these problems and yield greater sensitivity and specificity in the diagnosis of KIT-negative GISTs. ${ }^{19}$

In this study, the clinicopathological and molecular features of 26 KIT-negative GISTs were analyzed. Immunostaining of DOG1 and PKC- $\theta$ and mutational analysis for KIT and PDGFRA genes in KIT-negative GISTs were performed, and compared with KIT-positive GISTs.

\section{Materials and methods}

Cases consisted of 26 primary tumors having typical clinical and histological features of GISTs, but lacked KIT expression in formalin-fixed paraffinembedded tissues. Typical histological features of spindle cell-type GISTs were relatively uniform eosinophilic cells arranged in short fascicles or whorls with a pale eosinophilic cytoplasm, often with a fibrillary, syncytial appearance. GISTs of the

Table 1 Clinicopathological features in 26 KIT-negative gastrointestinal stromal tumors

\begin{tabular}{|c|c|c|c|c|c|c|c|}
\hline Case & Age (years)/gender & Location & Histology & Size $(\mathrm{cm})$ & Mitoses/50 HPF & Risk & Follow-up \\
\hline 1 & $55 / F$ & Stomach & Spindle & 5.5 & 2 & Low & No recur \\
\hline 2 & $57 / \mathrm{M}$ & Stomach & Epithelioid & 19.0 & 40 & High & Recur $^{a}$ \\
\hline 3 & $53 / \mathrm{F}$ & Stomach & Spindle & 2.9 & 1 & Very low & No recur \\
\hline 4 & $41 / \mathrm{M}$ & Stomach & Spindle & 2.5 & 1 & Very low & No recur \\
\hline 5 & $75 / \mathrm{M}$ & Stomach & Spindle & 0.8 & 0 & Very low & No recur \\
\hline 6 & $44 / \mathrm{M}$ & Stomach & Spindle & 1.1 & 0 & Very low & No recur \\
\hline 7 & $48 / \mathrm{M}$ & Stomach & Mixed & 4.5 & 2 & Very low & No recur \\
\hline 8 & $62 / \mathrm{M}$ & Stomach & Spindle & 3.8 & 1 & Very low & No recur \\
\hline 9 & $71 / \mathrm{F}$ & Stomach & Epithelioid & 10.0 & 2 & Low & No recur \\
\hline 10 & $59 / F$ & Stomach & Epithelioid & 4.0 & 1 & Low & No recur \\
\hline 11 & $29 / F$ & Stomach & Epithelioid & 3.5 & 15 & Moderate & Lost \\
\hline 12 & $70 / \mathrm{M}$ & Stomach & Epithelioid & 0.4 & 0 & Very low & No recur \\
\hline 13 & $65 / \mathrm{M}$ & Stomach & Epithelioid & 7.5 & 3 & Low & No recur \\
\hline 14 & $40 / \mathrm{M}$ & Stomach & Epithelioid & 9.5 & 6 & High & No recur \\
\hline 15 & $60 / \mathrm{M}$ & Stomach & Epithelioid & 4.0 & 4 & Very low & No recur \\
\hline 16 & $48 / \mathrm{M}$ & Stomach & Mixed & 5.8 & 6 & High & No recur \\
\hline 17 & $51 / \mathrm{M}$ & Stomach & Mixed & 5.5 & 9 & High & No recur \\
\hline 18 & $71 / \mathrm{F}$ & Stomach & Epithelioid & 2 & 7 & Very low & No recur \\
\hline 19 & $48 / \mathrm{F}$ & Stomach & Epithelioid & 8.0 & 70 & High & Recur $^{b}$ \\
\hline 20 & $63 / \mathrm{M}$ & Stomach & Spindle & 1.0 & 0 & Very low & No recur \\
\hline 21 & $63 / \mathrm{F}$ & Stomach & Spindle & 4.5 & 3 & Very low & No recur \\
\hline 22 & $52 / \mathrm{M}$ & Rectum & Spindle & 3.0 & 6 & High & No recur \\
\hline 23 & $72 / \mathrm{F}$ & Stomach & Spindle & 7.1 & 6 & High & No recur \\
\hline 24 & $62 / \mathrm{F}$ & Stomach & Spindle & 6.0 & 6 & High & No recur \\
\hline 25 & $41 / \mathrm{F}$ & Stomach & Spindle & 15.0 & 14 & High & Recur $^{b}$ \\
\hline 26 & $45 / \mathrm{M}$ & Stomach & Epithelioid & 11.0 & 2 & Moderate & No recur \\
\hline
\end{tabular}

${ }^{\mathrm{a} C a s e s}$ with recurrence and those that showed partial response to imatinib are given here.

${ }^{b}$ Cases with recurrence or metastasis before the era of imatinib are given here. 
epithelioid type were composed of round cells with a variably eosinophilic or clear cytoplasm. ${ }^{7}$

A total of 21 cases had undergone surgical resection at a single institute between 1999 and 2009 out of 754 GISTs (3\%), and 5 were from the consultation files of one of the authors (K-M Kim). Four of these KIT-negative tumors were reported in a previous study. ${ }^{19}$ Small biopsy specimens and tumors obtained after treatment with imatinib were excluded from this study. Pathological reports and hematoxylin and eosin-stained slides were reviewed in all cases, and estimated tumor characteristics included location, size, histological subtype, and mitotic count per 50 high power fields.

Sections of $4-\mu \mathrm{m}$ thickness were cut from paraffin blocks, followed by deparaffinization and rehydration. Immunohistochemistry was performed for KIT (polyclonal A4502, Dako, 1:250) in all cases without antigen retrieval, as described previously. ${ }^{22}$ DOG1 (clone K9, Leica Microsystems, Wetzlar, Germany, 1:200) was applied after heat-induced antigen retrieval in EDTA buffer at pH 8.0 for $1 \mathrm{~h} .^{5}$ PKC- $\theta$ (clone 27, BD Transduction Laboratories, 1:200) and
CD34 (clone QBEnd10, Dako, 1:100) immunostaining were also performed using the Leica Bond-Max System after antigen retrieval with Bond Epitope Retrieval Solution (Leica Microsystems). Diaminobenzidine was used as a chromogen. As control, 112 KIT-positive GISTs were used, and two 3-mm-sized cores were taken from representative areas for tissue microarray construction (Isu Abxis Co. Ltd, Seoul, Korea). Slides were scanned using ScanScope CS (Aperio Technologies, Vista, CA), and immunoreactivities were assessed by two pathologists (K-M Kim and G-H Kang) blinded to clinicopathological findings. The staining intensity of DOG1 and PKC- $\theta$ was graded as negative, weak, moderate, or strong. Cases were considered negative when $<10 \%$ tumor cells were stained. We considered cases to be positive only when $>10 \%$ tumor cells showed unequivocal immunoreactivity.

For comparison, 83 additional gastrointestinal mesenchymal tumors that often enter the differential diagnosis of KIT-negative GISTs, including mesenteric fibromatosis (47), schwannoma (6), leiomyoma (6), leiomyosarcoma (6), inflammatory
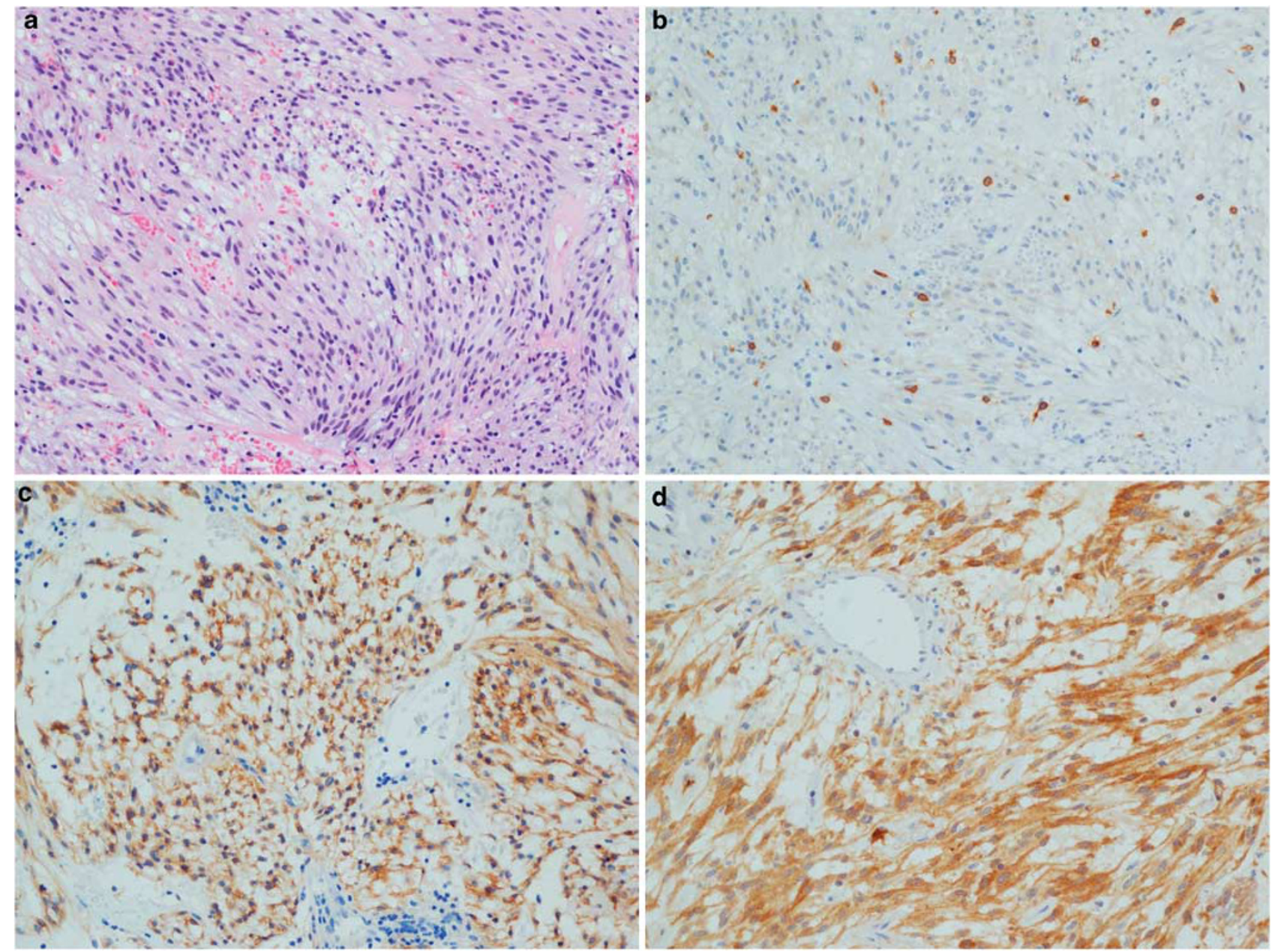

Figure 1 Spindle gastrointestinal stromal tumor (a) with negative KIT (b), positive DOG1 (c), and positive PKC- $\theta$ (d). 
fibroid polyp (6), malignant peripheral nerve sheath tumor (6), and melanoma (6), were also stained for DOG1 and PKC- $\theta$.

All 26 KIT-negative and 74 KIT-positive GISTs were analyzed for mutations in exons $9,11,13$, and 17 of KIT and in exons 12, 14, and 18 of the PDGRFA gene. DNA was extracted from $0.1 \%$ methylene bluestained $10-\mu \mathrm{m}$-thick sections under microscopy using a 20-G needle and proteinase $\mathrm{K}$ solution. PCR products were electrophoresed through $8 \%$ polyacrylamide gel with ethidium bromide to confirm correct amplification. The amplified products were purified, and both strands were directly sequenced using the BigDye Terminator v3.1 cycle sequencing kit, followed by analysis using an Applied Biosystems 3700 automated sequencer (Applied Biosystems, Foster City, CA).

Statistical analyses were carried out using SPSS 15.0 for Windows (SPSS Inc., Chicago, IL). Pearson's $\theta$ test or Fisher's exact test was used to examine the association between categorical variables, and McNemar's test was performed to assess the difference between paired proportions. A $P$-value $<0.05$ was considered statistically significant.

\section{Results}

\section{Clinical and Pathological Features of KIT-Negative GISTs}

The clinicopathological features of 26 KIT-negative GISTs are described in Table 1. There were 15 men and 11 women with ages ranging from 29 to 75 years (mean, 55.6). All tumors exhibited typical histological features of GISTs with complete loss of KIT expression by immunohistochemistry (Figures 1 and 2). All except one KIT-negative GIST were located in the stomach $(96 \%)$, and the remaining one in the rectum $(4 \%)$. The histological subtype was spindle in $12(46 \%)$, epithelioid in $11(42 \%)$, and mixed in 3 cases $(12 \%)$. The histological subclassification of spindle cell GISTs included seven hypercellular, three sclerosing, and two palisading and vacuolated types. Of epithelioid tumors, seven were hypercellular, two sclerosing, and two of the sarcomatous type. ${ }^{4}$ On the basis of tumor locations, sizes, and mitotic counts, 9 GISTs $(35 \%)$ were classified as high, $2(8 \%)$ as moderate, 4 $(15 \%)$ as low, and $11(42 \%)$ as being at a very low risk of aggressive behavior. ${ }^{2,23}$

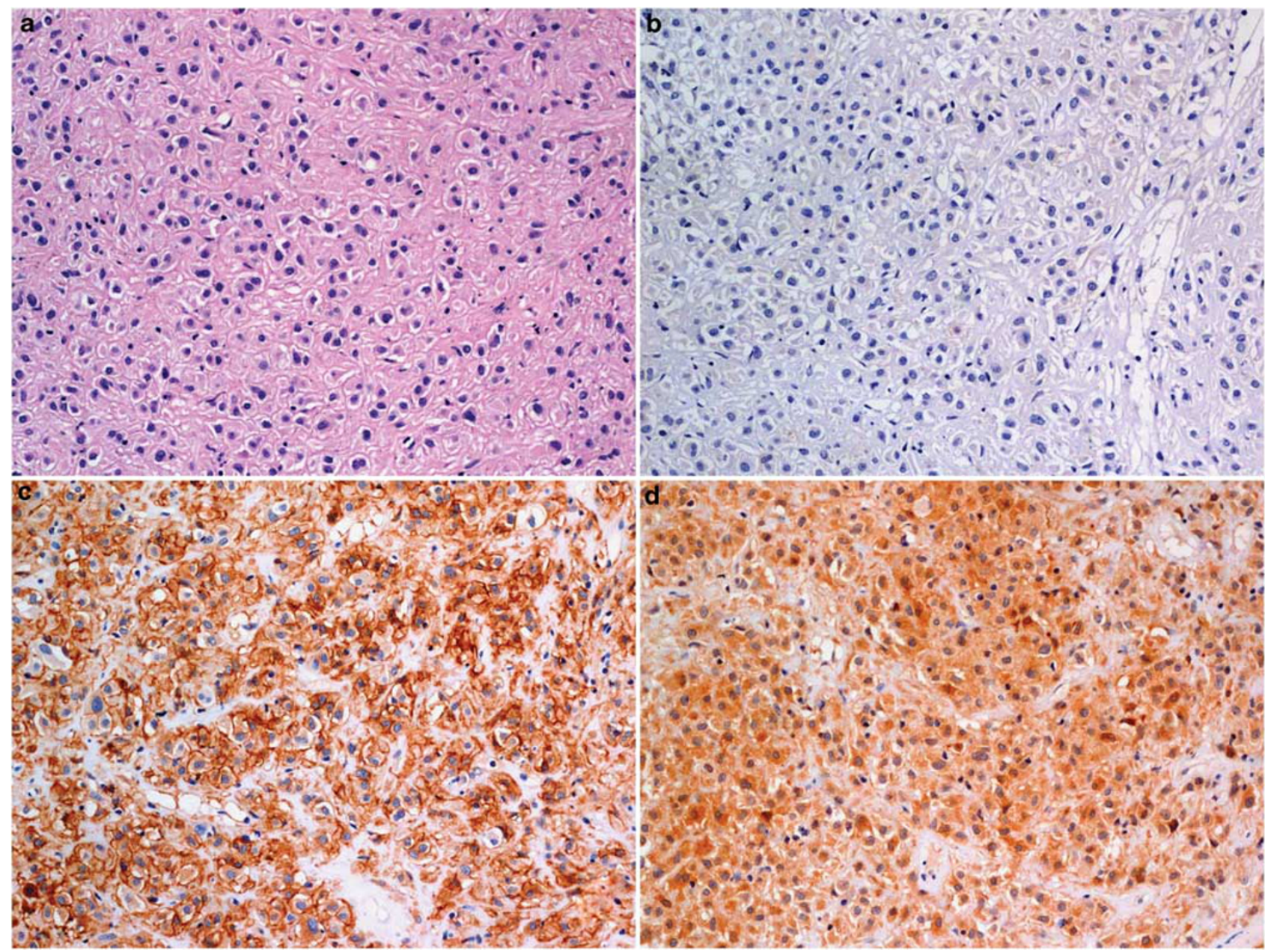

Figure 2 Epithelioid gastrointestinal stromal tumor (a) with negative KIT (b), positive DOG1 (c), and positive PKC- $\theta$ (d). 
Follow-up was available in 25 patients and local recurrence or metastasis was observed in 3 cases, initially classified as being at a high risk of aggressive behavior. Two patients had shown tumor progression before the imatinib era and died of disease. The remaining one patient had a partial response to imatinib during 5 years of follow-up.

\section{Immunohistochemistry and Mutation Analysis in 26 KIT-Negative GISTs}

Of 26 KIT-negative GISTs (92\%), 24 showed diffuse cytoplasmic and membranous staining for DOG1. The overall cytoplasmic and membranous staining intensity was strong in $12(46 \%)$, moderate in 9 $(35 \%)$, and weak in 3 cases (12\%) (Figure 3). Diffuse cytoplasmic positivity for PKC- $\theta$ was observed in 25 cases (96\%). The staining intensity was strong in 19 $(73 \%)$ and weak in the remaining 6 cases $(23 \%)$ (Figure 4). PKC- $\theta$ was diffusely positive in two GISTs ( $8 \%$ ) negative for both KIT and DOG1, but the staining intensity was weak in one of these cases. CD34 was positive in 20 KIT-negative GISTs (77\%). In two GISTs negative for both KIT and DOG1, one was focally positive for CD34 and the other was completely negative (Table 2).

In mesenchymal tumors other than GISTs, all mesenteric fibromatoses, inflammatory fibroid polyps, schwannomas, leiomyomas, malignant peripheral nerve sheath tumors, leiomyosarcomas, and melanomas were negative for DOG1. Two of six schwannomas (33\%) and malignant peripheral nerve sheath tumors $(33 \%)$ were focally positive for PKC- $\theta$. However, all mesenteric fibromatoses, inflammatory fibroid polyps, leiomyomas, leiomyosarcomas, and melanomas were completely negative for PKC- $\theta$.

On mutation analyses, 15 of 26 cases (58\%) harbored mutations in exon 18 of the PDGFRA gene, including 11 missense and 4 deletion mutations. One case $(4 \%)$ showed deletion mutation in exon 11 of the KIT gene. The remaining 10 GISTs (38\%) were wild type for KIT and PDGFRA genes (Table 2). Of the 10 tumors wild type on mutation analysis, DOG1 immunoreactivity was strong in 6, moderate and weak in 1 each, and completely negative in 2 cases. In these two DOG1-negative GISTs, PKC- $\theta$ was stained diffusely on whole tissue section with strong and weak intensities, respectively.
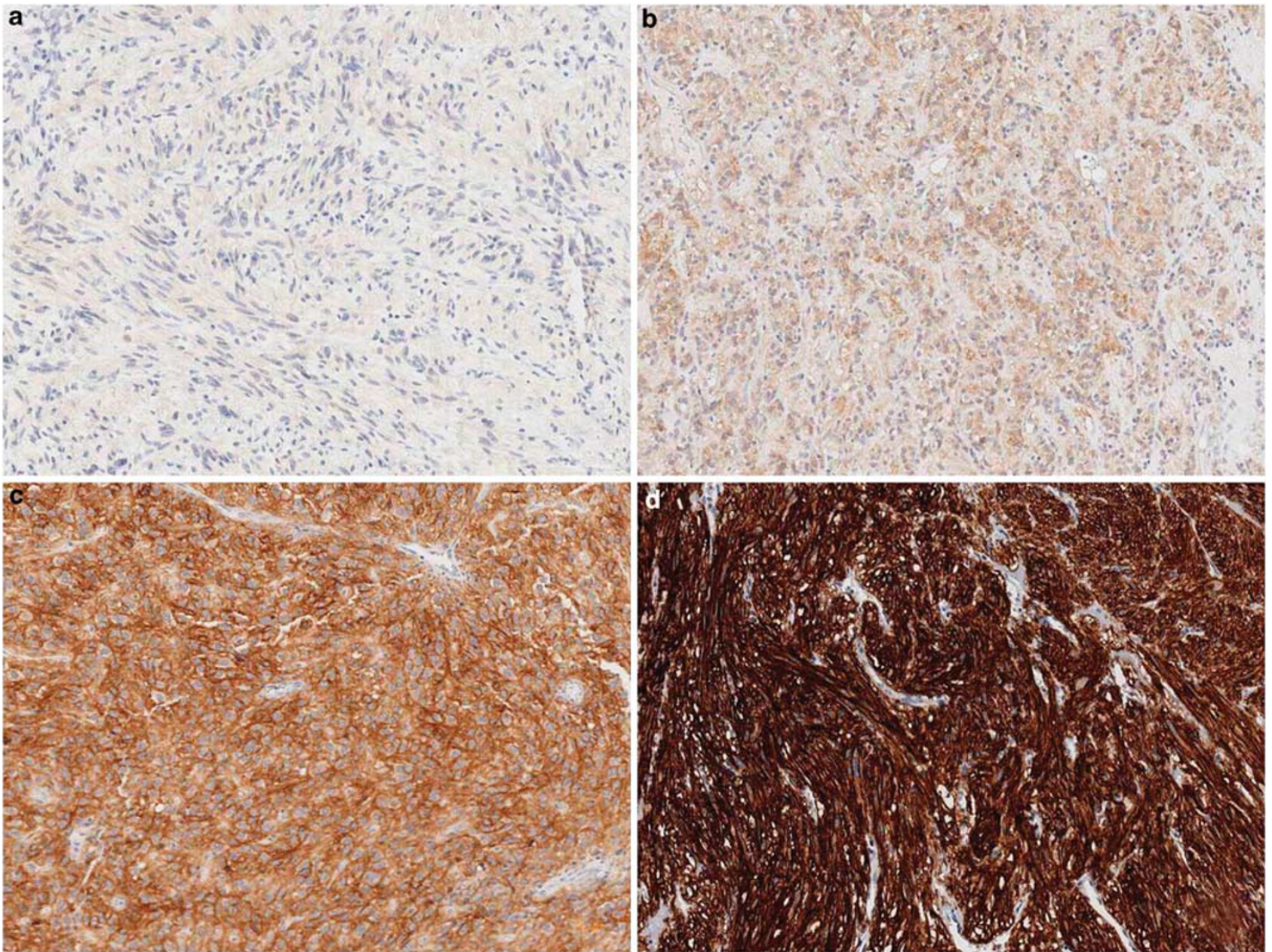

Figure 3 Spectrum of DOG1 immunoreactivity in KIT-negative gastrointestinal stromal tumors: negative (a), weak (b), moderate (c), and strong $(\mathbf{d})$. 
Immunohistochemistry and Mutation Analysis in 112 KIT-Positive GISTs

DOG1 was positive in 100 of 112 KIT-positive GISTs $(89 \%)$. The overall staining intensity was strong in $40(36 \%)$, moderate in $36(32 \%)$, and weak in 24 cases $(21 \%)$. A total of 93 (83\%) KIT-positive GISTs were positive for PKC- $\theta$, and the intensity was strong in 71 cases (63\%). CD34 was positive in 76 of 112 cases (68\%). Of 74 KIT-positive GISTs with mutation results, $52(70 \%)$ revealed KIT exon 11 mutations, including 30 deletion, 6 duplication, and 16 missense mutations. Overall, 10 cases (14\%) harbored KIT exon 9 mutations and $3(4 \%)$ had PDGFRA exon 18 mutations.

\section{Comparison Between KIT-Negative and KIT-Positive GISTs}

The clinicopathological features and immunohistochemical results of GISTs according to KIT expression are shown in Table 3. Among 26 KIT-negative and 74 KIT-positive tumors with mutation analyses, KIT mutations were detected in 17 of 39 GISTs $(44 \%)$ showing pure or mixed epithelioid morphology and in 46 of 61 tumors $(75 \%)$ with pure spindle cell type, the difference being statistically significant $(P=0.001)$.

Most KIT-negative GISTs occurred in the stomach compared with KIT-positive tumors (Fisher's exact test, $P<0.001)$, and were more likely to have an epithelioid morphology $(P=0.006)$. Wild-type KIT and PDGFRA gene status was significantly more common in KIT-negative GISTs than in KIT-positive tumors $(P=0.003)$. The concordance rate between DOG1 and PKC- $\theta$ expression was 89 and $83 \%$ in KIT-negative and KIT-positive GISTs, respectively. There was no significant difference between the expression of two markers in the KIT-negative and KIT-positive groups (McNemar's test, $P=1.00$ and $P=0.16$, respectively). The relationship of DOG1 and PKC- $\theta$ expression is described in Table 4. KITnegative GISTs were positive for either DOG1 or PKC- $\theta$, and six KIT-positive tumors (5\%) were negative for both markers.
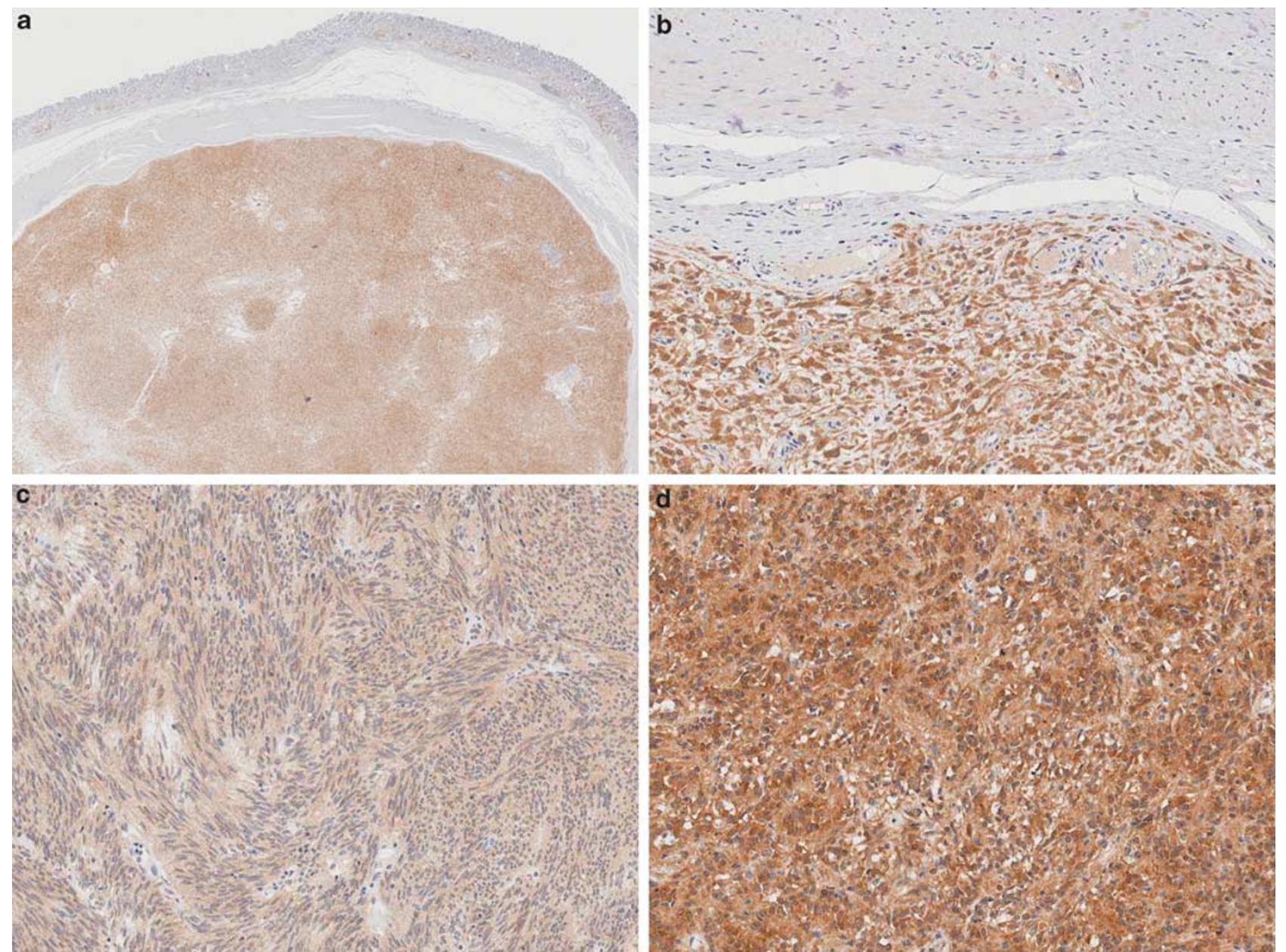

Figure 4 Diffuse PKC- $\theta$ positivity in a KIT-negative gastrointestinal stromal tumor. Diffuse cytoplasmic positivity is present in tumor cells compared with negative background staining in the gastric mucosa and muscularis propria (a). Higher magnification of panel a showing unequivocal positivity in tumor cells with negative background staining (b). PKC- $\theta$ staining intensity in our series is either weak (c) or diffusely strong (d) in tumor cells. 
Table 2 Immunohistochemical staining and mutation results in 26 KIT-negative gastrointestinal stromal tumors

\begin{tabular}{|c|c|c|c|c|c|}
\hline \multirow[t]{2}{*}{ Case } & \multicolumn{3}{|c|}{ Immunohistochemistry } & \multirow[t]{2}{*}{ Mutation } & \multirow{2}{*}{$\begin{array}{l}\text { Imatinib } \\
\text { sensitive }\end{array}$} \\
\hline & DOG1 & $P K C-\theta$ & $C D 34$ & & \\
\hline 1 & Negative & Strong & Focal & Wild type & Yes \\
\hline 2 & Moderate & Weak & Diffuse & KIT exon 11 deletion (KPMYEVQ550-556) & Yes \\
\hline 3 & Strong & Strong & Diffuse & Wild type & Yes \\
\hline 4 & Moderate & Strong & Diffuse & PDGFRA exon 18 missense (D842V) & No \\
\hline 5 & Strong & Weak & Diffuse & Wild type & Yes \\
\hline 6 & Strong & Weak & Diffuse & Wild type & Yes \\
\hline 7 & Weak & Strong & Focal & PDGFRA exon 18 missense (D842V) & No \\
\hline 8 & Weak & Strong & Diffuse & PDGFRA exon 18 missense (D842H, D846A) & NR \\
\hline 9 & Moderate & Strong & Diffuse & PDGFRA exon 18 missense(D842Y) & Yes \\
\hline 10 & Moderate & Strong & Negative & PDGFRA exon 18 missense (D842V) & No \\
\hline 11 & Strong & Strong & Negative & PDGFRA exon 18 missense (D842V) & No \\
\hline 12 & Weak & Strong & Focal & Wild type & Yes \\
\hline 13 & Moderate & Strong & Diffuse & PDGFRA exon 18 missense (D842V) & No \\
\hline 14 & Moderate & Weak & Diffuse & Wild type & Yes \\
\hline 15 & Strong & Strong & Diffuse & PDGFRA exon 18 missense (D842V) & No \\
\hline 16 & Strong & Negative & Negative & PDGFRA exon 18 missense (D842V) & No \\
\hline 17 & Moderate & Strong & Negative & PDFGRA exon 18 deletion (MHDS844-847) & NR \\
\hline 18 & Strong & Strong & Negative & PDGFRA exon 18 deletion (DIMH842-845) & Yes \\
\hline 19 & Strong & Strong & Diffuse & PDGFRA exon 18 missense (D842V) & No \\
\hline 20 & Strong & Strong & Diffuse & PDGFRA exon 18 deletion (MHDS844-847) & NR \\
\hline 21 & Strong & Strong & Diffuse & Wild type & Yes \\
\hline 22 & Strong & Strong & Diffuse & Wild type & Yes \\
\hline 23 & Strong & Strong & Diffuse & Wild type & Yes \\
\hline 24 & Moderate & Strong & Diffuse & PDGFRA exon 18 deletion (MHDS844-847) & NR \\
\hline 25 & Negative & Weak & Negative & Wild type & Yes \\
\hline 26 & Moderate & Weak & Focal & PDGFRA exon 18 missense (D846Y) & Yes \\
\hline
\end{tabular}

PDGFRA, platelet-derived growth factor receptor- $\alpha$; NR, not reported.

\section{Discussion}

In this study, we present a series of 26 KIT-negative GISTs in which DOG1 and PKC- $\theta$ were proven extremely valuable for making a definitive diagnosis. As reported previously, KIT-negative GISTs were more likely to have epithelioid morphology and were commonly located in the stomach. ${ }^{3-5}$ In concordance with previous studies, ${ }^{24,25}$ epithelioid cell morphology was commonly observed in GISTs lacking KIT mutations. KIT-negative and KIT-positive GISTs shared the common clinicopathological features, including patients' age, gender, and the risk of aggressive behavior.

We confirmed that the DOG1 antibody, clone K9, works well on paraffin-embedded tissues and is highly expressed in GISTs irrespective of KIT expression. DOG1 was also positive in $89 \%$ of KIT and PDGFRA wild-type GISTs, and the expression was not related to mutational status. As described previously, cytoplasmic staining was more pronounced in spindle cells, whereas epithelioid cells showed prominent membranous staining. ${ }^{8}$ It has been demonstrated that clone K9 and monoclonal DOG1.1 are expressed in 95 and $87 \%$ of GISTs, respectively, indicating that the new clone K9 antibody may be more sensitive. ${ }^{5,17}$ The positivity of DOG1 in KIT-negative GISTs was variable according to the antibodies used: with DOG1.1, the positivity is $36 \%{ }^{8}$ and with $\mathrm{K} 9$, the positivity is
$50-76 \% .^{5,26}$ In our study, with clone K9 and whole paraffin tissue blocks, $92 \%$ of KIT-negative GISTs were positive for DOG1. This difference may be caused by the type of tissue sections used for immunohistochemistry. We used whole paraffin tissue block instead of multi-tumor blocks containing 5-60 tumors ${ }^{5}$ or tissue microarrays. ${ }^{27}$ These limit the amount of tumor tissues analyzed for immunoreactivity and does not reflect that daily routine practice in which whole tissue blocks are used for immunohistochemical workup. Miettinen et $a l^{5}$ also reported that DOG1 (clone K9) reacted slightly better in gastric epithelioid GISTs than in KIT, and that KIT was slightly more sensitive than DOG1 in intestinal GISTs. ${ }^{5}$ In our analyses, the positivity for KIT was higher in GISTs of non-gastric locations, and the expression was more frequently observed in spindle cell-type GISTs. However, DOG1 immunoreactivity was not significantly associated with tumor location and histological subtype.

Some authors have noted that PKC- $\theta$ is not a useful diagnostic marker for GISTs because of high background staining and limited reproducibility. ${ }^{2,5,8,21}$ However, we could get a satisfactory signal-to-noise ratio at a dilution of 1:200 with antigen retrieval (PKC- $\theta$, clone 27). PKC- $\theta$ is also reported to be expressed in schwannoma, malignant peripheral nerve sheath tumor, and Ewing's sarcoma/primitive neuroectodermal tumor. ${ }^{19,28}$ In our series, all cases of mesenteric fibromatosis, 
Table 3 Clinicopathological features and results of immunohistochemistry according to KIT expression

\begin{tabular}{|c|c|c|c|}
\hline & $\begin{array}{c}\text { KIT-negative } \\
(\mathrm{n}=26)\end{array}$ & $\begin{array}{c}\text { KIT-positive } \\
(\mathrm{n}=112)\end{array}$ & $\mathrm{P}$-value \\
\hline $\begin{array}{l}\text { Mean age } \\
\text { (range) }\end{array}$ & $\begin{array}{c}55.6 \text { years } \\
(29-75)\end{array}$ & $\begin{array}{c}55.5 \text { years } \\
(26-88)\end{array}$ & NS \\
\hline \multicolumn{4}{|l|}{ Gender } \\
\hline Male & 15 & 54 & NS \\
\hline Female & 11 & 58 & \\
\hline \multicolumn{4}{|l|}{ Location } \\
\hline Stomach & 25 & 60 & $<0.001^{\mathrm{a}}$ \\
\hline Small & 0 & 46 & \\
\hline intestine & & & \\
\hline Esophagus & 0 & 2 & \\
\hline Rectum & 1 & 1 & \\
\hline Mesentery & 0 & 3 & \\
\hline \multicolumn{4}{|l|}{ Histology } \\
\hline Spindle & 12 & 83 & $0.006^{\mathrm{b}}$ \\
\hline Epithelioid & 11 & 10 & \\
\hline Mixed & 3 & 19 & \\
\hline \multicolumn{4}{|l|}{ Risk } \\
\hline High & 9 & 50 & NS \\
\hline Moderate & 2 & 14 & \\
\hline Very low/low & 15 & 48 & \\
\hline \multicolumn{4}{|l|}{ Mutation } \\
\hline $\begin{array}{l}\text { KIT exon } 9 \\
\text { deletion }\end{array}$ & 0 & 1 & $\begin{array}{c}<0.001^{\mathrm{c}} \\
0.003^{\mathrm{d}}\end{array}$ \\
\hline $\begin{array}{l}\text { KIT exon } 9 \\
\text { duplication }\end{array}$ & 0 & 9 & \\
\hline $\begin{array}{l}\text { KIT exon } 11 \\
\text { deletion }\end{array}$ & 1 & 30 & \\
\hline $\begin{array}{l}\text { KIT exon } 11 \\
\text { duplication }\end{array}$ & 0 & 6 & \\
\hline $\begin{array}{l}\text { KIT exon } 11 \\
\text { missense }\end{array}$ & 0 & 16 & \\
\hline $\begin{array}{l}\text { PDGRFA exon } \\
18 \text { deletion }\end{array}$ & 4 & 0 & \\
\hline PDGRFA exon & 11 & 3 & \\
\hline 18 missense & & & \\
\hline Wild type & 10 & 9 & \\
\hline Sum & 26 & 74 & \\
\hline \multicolumn{4}{|l|}{ DOG1 } \\
\hline Negative & 2 & 12 & NS \\
\hline Weak & 3 & 24 & \\
\hline Moderate & 9 & 36 & \\
\hline Strong & 12 & 40 & \\
\hline \multicolumn{4}{|l|}{$P K C-\theta$} \\
\hline Negative & 1 & 19 & NS \\
\hline Weak & 6 & 22 & \\
\hline Strong & 19 & 71 & \\
\hline \multicolumn{4}{|l|}{ Total } \\
\hline & 26 & 112 & \\
\hline
\end{tabular}

NS, not significant.

${ }^{\mathrm{a}}$ Gastric vs non-gastric location and Fisher's exact test are represented here.

${ }^{\mathrm{b}}$ Spindle $v s$ epithelioid and mixed types are represented here.

${ }^{\mathrm{C}}$ PDGRFA mutations vs $c$-kit mutations and wild type, and Fisher's exact test are represented here.

${ }^{\mathrm{d}}$ Wild type vs PDGFRA and c-kit mutations are represented here. inflammatory fibroid polyp, leiomyoma, leiomyosarcoma, and melanoma, which mimic GIST and are the usual considerations in differential diagnosis, were completely negative for PKC- $\theta .{ }^{19,21}$ In previous studies with KIT-negative GISTs, $3-4 \%$ was negative for the new DOG1 antibody. ${ }^{5,27}$ PKC- $\theta$ was positive in two cases $(8 \%)$ and completely negative for both KIT and DOG1 in this study, which was helpful in validating these cases as GISTs.

In this study, both DOG1 and PKC- $\theta$ were expressed more frequently in KIT-negative GISTs than in KIT-positive GISTs, but this difference was not statistically significant. These findings are consistent with previous observations that DOG1 shows higher prevalence of positivity in gastric epithelioid GISTs, which are often KIT negative. ${ }^{5}$ PKC- $\theta$ positivity in KIT-negative GISTs has also been reported previously. ${ }^{19,20}$

We also found that $58 \%$ of KIT-negative GISTs in our series harbored mutations in exon 18 of PDGFRA, leading to a substitution of valine for aspartic acid (D842V), known to be imatinib mesylate (Gleevec) resistant. ${ }^{10,29}$ The KIT mutation was present in one KIT-negative tumor (4\%). In previous studies on KIT-negative GISTs, the PDGFRA mutation $(\mathrm{D} 842 \mathrm{~V})$ and the KIT mutation were observed in $30-40 \%$ and $0-16 \%$ of cases, respectively. ${ }^{9,20}$ Mutations found in $15 \%$ of KIT-negative GISTs were PDGFRA exon 18 deletions, which are imatinib sensitive. ${ }^{1}$ PDGFRA exon 18 deletion mutations have been reported in $7-17 \%$ of KIT-negative tumors, and our results are consistent with previous reports. ${ }^{3,8,30}$ Wild-type KIT and PDGFRA status was observed in $38 \%$ of cases. Medeiros et $\mathrm{al}^{3}$ and Lee et $a 2^{21}$ reported that $16-70 \%$ of KIT-negative tumors were wild type. One of our patients (case 11) was a 29-year-old woman with an epithelioid gastric GIST, which raised the possibility of familial or idiopathic multi-tumor syndrome. ${ }^{31}$ However, the presence of a solitary tumor in the absence of a family history, negative chest roentgenogram, and the absence of any adrenal mass ruled out the possibility of Carney triad.

Recently, it has been shown that a subset of GISTs without KIT or PDGFRA mutations are also responsive to imatinib. ${ }^{2}$ Although the PDGFRA D842V mutation is intrinsically imatinib resistant, more than two-thirds of KIT-negative tumors are potentially imatinib sensitive. ${ }^{3,10,32}$ Moreover, imatinib is still regarded as the first-line therapy for advanced GISTs, including those with PDGFRA D842V mutations. ${ }^{1}$ In our 26 KIT-negative GISTs, 3 patients showed recurrence of disease and 1 patient with KIT mutation had a partial response to imatinib therapy. Therefore, it is important for pathologists and oncologists to be aware that, in the context of an otherwise typical morphology, a diagnosis of GIST should not be precluded on the basis of negative staining for KIT and such patients should not, a priori, be denied imatinib therapy. ${ }^{9}$ For the diagnosis of KIT-negative GISTs with a wild-type 
Table 4 The relationship between expression of DOG1 and PKC- $\theta$ in gastrointestinal stromal tumors

\begin{tabular}{|c|c|c|c|c|c|c|c|c|c|}
\hline \multirow[t]{3}{*}{ DOG1 } & \multicolumn{3}{|c|}{26 KIT (-) GISTs } & \multicolumn{3}{|c|}{112 KIT (+) GISTs } & \multicolumn{3}{|c|}{ All 138 GISTs } \\
\hline & \multicolumn{2}{|c|}{$P K C-\theta$} & \multirow[t]{2}{*}{$\mathrm{P}$-value ${ }^{\mathrm{a}}$} & \multicolumn{2}{|c|}{$P K C-\theta$} & \multirow[t]{2}{*}{$\mathrm{P}$-value $\mathrm{a}^{\mathrm{a}}$} & \multicolumn{2}{|c|}{$P K C-\theta$} & \multirow[t]{2}{*}{$\mathrm{P}$-value } \\
\hline & Negative & Positive & & Negative & Positive & & Negative & Positive & \\
\hline Negative & 0 & 2 & 1.00 & 6 & 6 & 0.16 & 6 & 8 & 0.28 \\
\hline Positive & 1 & 23 & & 13 & 87 & & 14 & 110 & \\
\hline
\end{tabular}

${ }^{\mathrm{a}}$ McNemar's test is represented here.

mutation, DOG1 and PKC- $\theta$ immunostaining will be helpful in solving the diagnostic difficulties. PKC- $\theta$ was positive in two cases which were completely negative for both KIT and DOG1. Therefore, when a pathologist confronts KIT-negative GIST-like lesions, PKC- $\theta$ is extremely useful in diagnosing GISTs with certainty. The initial workup for possible GISTs should include KIT and DOG1, and in rare cases, negative for both KIT and DOG1, PKC- $\theta$ can be used as an important ancillary stain.

In summary, we report that a subset of GISTs lack KIT expression, although they show overlapping clinical, histopathological, and molecular features with their KIT-positive counterparts. DOG1 and PKC- $\theta$ are highly expressed in both KIT-negative and KIT-positive GISTs. Combined immunostaining for DOG1 and PKC- $\theta$ can be used as an important diagnostic tool in the diagnosis of KIT-negative GISTs, even those that are wild type for KIT and PDGFRA on mutation analysis.

\section{Disclosure/conflict of interest}

The authors declare no conflict of interest.

\section{References}

1 Debiec-Rychter M, Wasag B, Stul M, et al. Gastrointestinal stromal tumours (GISTs) negative for KIT (CD117 antigen) immunoreactivity. J Pathol 2004;202:430-438.

2 Liegl-Atzwanger B, Fletcher JA, Fletcher CD. Gastrointestinal stromal tumors. Virchows Arch 2010;456: 111-127.

3 Medeiros F, Corless CL, Duensing A, et al. KIT-negative gastrointestinal stromal tumors: proof of concept and therapeutic implications. Am J Surg Pathol 2004;28: 889-894.

4 Miettinen M, Sobin LH, Lasota J. Gastrointestinal stromal tumors of the stomach: a clinicopathologic, immunohistochemical, and molecular genetic study of 1765 cases with long-term follow-up. Am J Surg Pathol 2005;29:52-68.

5 Miettinen M, Wang ZF, Lasota J. DOG1 antibody in the differential diagnosis of gastrointestinal stromal tumors: a study of 1840 cases. Am J Surg Pathol 2009;33: 1401-1408.
6 Pauls K, Merkelbach-Bruse S, Thal D, et al. PDGFRalpha- and c-kit-mutated gastrointestinal stromal tumours (GISTs) are characterized by distinctive histological and immunohistochemical features. Histopathology 2005;46:166-175.

7 Fletcher CD, Berman JJ, Corless C, et al. Diagnosis of gastrointestinal stromal tumors: a consensus approach. Hum Pathol 2002;33:459-465.

8 Liegl B, Hornick JL, Corless CL, et al. Monoclonal antibody DOG1.1 shows higher sensitivity than KIT in the diagnosis of gastrointestinal stromal tumors, including unusual subtypes. Am J Surg Pathol 2009;33: 437-446.

9 Corless CL, Fletcher JA, Heinrich MC. Biology of gastrointestinal stromal tumors. J Clin Oncol 2004;22: 3813-3825.

10 Heinrich MC, Corless CL, Demetri GD, et al. Kinase mutations and imatinib response in patients with metastatic gastrointestinal stromal tumor. J Clin Oncol 2003;21:4342-4349.

11 Demetri GD, von Mehren M, Blanke CD, et al. Efficacy and safety of imatinib mesylate in advanced gastrointestinal stromal tumors. N Engl J Med 2002;347: 472-480.

12 Wardelmann E, Buttner R, Merkelbach-Bruse S, et al. Mutation analysis of gastrointestinal stromal tumors: increasing significance for risk assessment and effective targeted therapy. Virchows Arch 2007;451:743-749.

13 Demetri GD, van Oosterom AT, Garrett CR, et al. Efficacy and safety of sunitinib in patients with advanced gastrointestinal stromal tumour after failure of imatinib: a randomised controlled trial. Lancet 2006;368:1329-1338.

14 Debiec-Rychter M, Cools J, Dumez H, et al. Mechanisms of resistance to imatinib mesylate in gastrointestinal stromal tumors and activity of the PKC412 inhibitor against imatinib-resistant mutants. Gastroenterology 2005;128:270-279.

15 West RB, Corless CL, Chen X, et al. The novel marker, DOG1, is expressed ubiquitously in gastrointestinal stromal tumors irrespective of KIT or PDGFRA mutation status. Am J Pathol 2004;165:107-113.

16 Katoh M, Katoh M. FLJ10261 gene, located within the CCND1-EMS1 locus on human chromosome 11q13, encodes the eight-transmembrane protein homologous to C12orf3, C11orf25 and FLJ34272 gene products. Int J Oncol 2003;22:1375-1381.

17 Espinosa I, Lee CH, Kim MK, et al. A novel monoclonal antibody against DOG1 is a sensitive and specific marker for gastrointestinal stromal tumors. Am J Surg Pathol 2008;32:210-218. 
18 Duensing A, Joseph NE, Medeiros F, et al. Protein kinase $\mathrm{C}$ theta (PKCtheta) expression and constitutive activation in gastrointestinal stromal tumors (GISTs). Cancer Res 2004;64:5127-5131.

19 Kim KM, Kang DW, Moon WS, et al. PKCtheta expression in gastrointestinal stromal tumor. Mod Pathol 2006;19:1480-1486.

20 Motegi A, Sakurai S, Nakayama H, et al. PKC theta, a novel immunohistochemical marker for gastrointestinal stromal tumors (GIST), especially useful for identifying KIT-negative tumors. Pathol Int 2005;55: 106-112.

21 Lee HE, Kim MA, Lee HS, et al. Characteristics of KITnegative gastrointestinal stromal tumours and diagnostic utility of protein kinase $\mathrm{C}$ theta immunostaining. J Clin Pathol 2008;61:722-729.

22 Hornick JL, Fletcher CD. Immunohistochemical staining for KIT (CD117) in soft tissue sarcomas is very limited in distribution. Am J Clin Pathol 2002;117: 188-193.

23 Miettinen M, Lasota J. Gastrointestinal stromal tumors: pathology and prognosis at different sites. Semin Diagn Pathol 2006;23:70-83.

24 Wardelmann E, Neidt I, Bierhoff E, et al. c-kit mutations in gastrointestinal stromal tumors occur preferentially in the spindle rather than in the epithelioid cell variant. Mod Pathol 2002;15: 125-136.

25 Heinrich MC, Rubin BP, Longley BJ, et al. Biology and genetic aspects of gastrointestinal stromal tumors:
KIT activation and cytogenetic alterations. Hum Pathol 2002;33:484-495.

26 Lopes LF, West RB, Bacchi LM, et al. DOG1 for the diagnosis of gastrointestinal stromal tumor (GIST): comparison between 2 different antibodies. Appl Immunohistochem Mol Morphol 2010;18:333-337.

27 Lopes LF, Bacchi CE. Imatinib treatment for gastrointestinal stromal tumour (GIST). J Cell Mol Med 2010;14:42-50.

28 Kang GH, Kim KM, Park CK, et al. PKC-theta expression in Ewing sarcoma/primitive neuroectodermal tumour and malignant peripheral nerve sheath tumour. Histopathology 2009;55:368-369.

29 Debiec-Rychter M, Dumez H, Judson I, et al. Use of cKIT/PDGFRA mutational analysis to predict the clinical response to imatinib in patients with advanced gastrointestinal stromal tumours entered on phase I and II studies of the EORTC Soft Tissue and Bone Sarcoma Group. Eur J Cancer 2004;40:689-695.

30 Miselli F, Millefanti C, Conca E, et al. PDGFRA immunostaining can help in the diagnosis of gastrointestinal stromal tumors. Am J Surg Pathol 2008;32: 738-743.

31 Agaimy A, Hartmann A. Hereditary and non-hereditary syndromic gastointestinal stromal tumours. Pathologe 2010;31:430-437.

32 Corless CL, Schroeder A, Griffith D, et al. PDGFRA mutations in gastrointestinal stromal tumors: frequency, spectrum and in vitro sensitivity to imatinib. J Clin Oncol 2005;23:5357-5364. 